\title{
Configurable Process Models for the Swedish Public Sector
}

\author{
Carl-Mikael Lönn, Elin Uppström, Petia Wohed, and Gustaf Juell-Skielse \\ Department of Computer and Systems Sciences, Stockholm University, Sweden \\ $\{\mathrm{cml}$, elinu, petia,gjs\}@dsv.su.se
}

\begin{abstract}
Process orientation and e-services have become essential in revitalizing local government. Although most municipalities offer similar services there is little reuse of e-services or underlying process models among municipalities. Configurable process models represent a promising solution to this challenge by integrating numerous variations of a process in one general model. In this study, design science is used to develop a configurable process model to capture the variability of a number of different processes. The results include a validated configurable process model for social services, a benefits analysis and directions for future development. Although the results are perceived useful by municipal officials, there are several challenges to be met before the benefits of configurable process models are fully utilized.
\end{abstract}

Keywords: Configurable process models, public administration processes, e-services, e-government.

\section{Introduction}

Local governments in Sweden are responsible for a large share of public services. Care for elderly and disabled account for almost $30 \%$ of municipal spending. To meet the challenges arising with an aging population [19], municipalities are working on (i) streamlining administration associated with provision of the public services and (ii) developing e-services. Important goals with the development of e-services is to provide $24 / 7$ accessibility, offer self-service capabilities to citizens and increase transparency between citizens and municipalities. This is made possible by the provision of e-forms and automated, positive, criteria-based decisions where feedback and decisions are electronically communicated almost instantly. To deal with both (i) and (ii) above, we have been involved in process analyses carried out within a project (OST) at the social services administration at Järfälla municipality [9]. Järfälla is one of 290 Swedish municipalities and is an average sized municipality situated $20 \mathrm{~km}$ northwest of Stockholm.

During the ÖST (Open Social e-Services) project three open social e-services for applications of emergency phone, companion and part time successor services were developed. For the development of each service a thorough analysis of the underlying process was performed with officials from the municipality. During the process analysis a large overlap between the three processes was observed. 

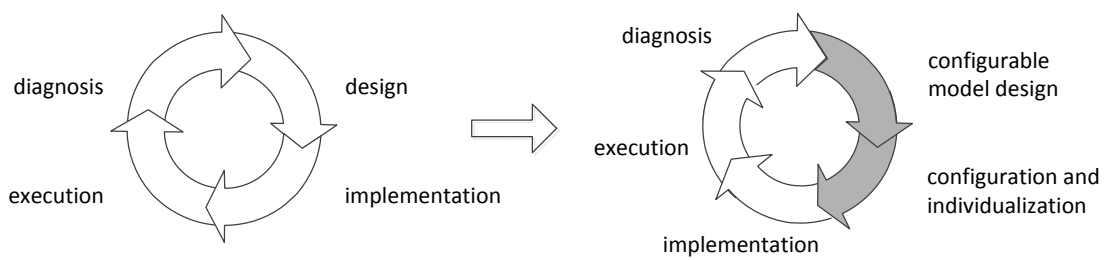

Fig. 1. The configurable process model life cycle (Source: [15])

To capture the similarities and keep the differences, we postulated that a configurable process model will provide a model at a suitable level of abstraction.

A configurable process model is a general process model that integrates a number of process variants. Decisions regarding variation points in the model depends on requirements of the organization and does not take into account data values that will be available when the process is executed [10]. The configurable process model also maintains domain data on how the individual variants can be derived from the general model. Such derivation is typically called individualization (or configuration) and is done in design time, a priory deployment and run-time, by splitting the PAIS design phase into a configurable model design phase and a configuration and individualization phase as seen in Figure 1.

Configurable models are suitable for the creation of reference models, i.e. general models that aim at capturing processes specific for certain domains. Configurable models are also applicable when a process is with small variation implemented in organizations with different geographical locations where the variations reflect cultural, legislative, or other differences. The situation in the public sector is similar where comparable processes are applied within different administrative regions in a country.

The main point with a configurable process modeling approach is to support process variability. This can be contrasted to the provision of standard solutions aimed at covering the needs of everyone. Within the ÖST project we encountered similar processes and decided to explore the benefits of considering these processes as variants of a general process. Therefore we applied a configurable process modeling approach. In particular we utilized the approach by Gottschalk et al. 4. Here we report our experiences from this work. Our study is similar to the study reported in [5. The similarity lies in the utilization of the same technology for creating configurable models. The difference lies in the way the technology is applied. While [5] creates a configurable model for one and the same process running at different municipalities, we create a configurable model based on three different processes sharing a portion of similarities. By doing so we envisage the following benefits.

1. Provide a comprehensive process model describing the work routines associated with application handling for social services. This general model will serve as a basis for shared understanding by the stakeholders. It will unite the commonalities and at the same time preserve the differences between the considered processes. 
2. Offer support for individualization. From the configurable model different individual models capturing the specific processes can easily be produced. To support the individualization we have build a domain model summarizing the knowledge on the differences of the models. In this way the configurable model will be individualized by its stakeholders (without any process modeling training).

3. Simplify process change management. General changes, e.g. changes reflecting updates in a law, can be applied on the configurable model, which makes model maintenance easier. Instead of introducing a change into three or several similar models (running the risk of introducing errors or loosing consistency) the change is introduced in one place only, i.e. the general model.

4. Increase process applicability. Even if we are currently focusing at the needs of one municipality, the configurable model may potentially be beneficial for the other municipalities in the country (as is or after some minor modifications further turning it to a yet more general solutions).

The paper is structured as follows. Section 2 presents the method of our work. Section 3 gives some background knowledge to the tool we have used, i.e. YAWL. Section 4 presents the configurable process model and the domain model we have developed. Section 5 discusses the evaluation of the results. Section 6 presents some challenges we met and how they were tackled. Section 7 presents directions for future work and Section 8 concludes the paper.

\section{Method}

We have followed the Design Science research approach [7]. Using the vocabulary of Hevner et al. 7], the artefact developed during our work is an instantiation, i.e. a proof-of-concept configurable model for applications of social services in the Swedish public sector. To ensure research rigor, we utilize the configurable approach defined in Gottschalk et al. 4 and its implementation in the YAWL environment [3], which we will refer to as C-YAWL. Some other approaches for dealing with variability within business process management have been developed for different process modeling notations, e.g., UML Activity Diagram [1814, EPC [16, or notation independent approaches such as PROVOP [6]. We selected C-YAWL because: (i) it is an executable environment. This feature makes it easier to demonstrate and test practical implications and methodology to users that are unfamiliar with process modeling 4]; (ii) The C-YAWL environment provides model verification functionality; (iii) it is open-source, which is essential for spreading the results to the other municipalities in the country and (iv) the YAWL notation was earlier used in the project resulting in a number of process models already being documented in YAWL and the familiarity of the municipal officials with the notation.

The development was carried out iteratively. First the three processes models, developed in earlier phases of the project, were integrated to a configurable model. Then the solution was validated through two workshops in which four 
municipal officials took part. During the first workshop, the model was presented and discussed with the business users, who were also asked to annotate the solution with any presumptive improvements (which they also did). Based on the input from the users, the model was improved and revisited during the second workshop.

The results were evaluated through a combination of analytical and observational evaluation methods [7. The analytical evaluation was done trough the verification functionality in YAWL 8, i.e. we ensured that the produced configurable model is sound. The observational evaluation was carried out through a case study [13. During the case study the quality of the model (i.e. how well it captures the domain under consideration) and the generality of the model were studied by exploring the model's capability for capturing a fourth process. When exploring the generality of the model, the postulated benefits 1-3 from Section 1 were studied.

The case study was carried out through four seminars and one workshop. The four seminars were attended by a working group containing five municipal officials, three customer relationship officers and two financial assistants, representing both the elderly and the handicapped unit at the municipality. During the seminars the configurable model for the three services was extended to cover the fourth service for the control-flow and resource perspectives. Then the data perspective was analyzed and documented. After this, the configuration points were presented and validated. During the workshop, the solution created in the seminars was presented to a broader audience. Initially, the workshop was scheduled to be attended by all municipal officials dealing with applications for social services (i.e. 25-30 employees), but due to unexpected external reasons which we could not influence, the workshop was attended by nine officials only. Among the participants were two managers for the elderly and handicapped administration, one program manager from the handicapped unit, one representative from the IT department, three of the officials from the working group (i.e. customer relationship officers and financial assistants), and the two project managers for the project.

\section{Background}

\subsection{The C-YAWL Notation}

In YAWL [8] a number of constructs are used to create workflow specifications. Figure 2 shows the relevant constructs for this work. These are divided into conditions, tasks and flows. Start and end conditions indicate the beginning and the end of a process. Tasks might have decorators. The decorators are used to capture joins and splits of the control flow. There are three types of decorators - AND, XOR and OR decorators - each coming in two variants, join and split. We have used colors to show the different roles responsible for the execution of the tasks.

C-YAWL [8] introduces the concept of port, i.e. a possible incoming or outgoing path of a task. For instance a XOR-join task with two incoming flows have 


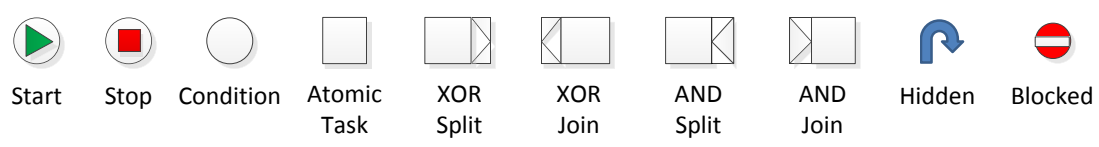

Fig. 2. The C-YAWL notation

two ports associated to it, one for each alternative paths enabling the task, while an AND-join task have one incoming port associated to it. C-YAWL contains in addition two settings, blocked and hidden, that may be used on the ports. Hidden on an incoming port indicates that the relevant task will be skipped and the flow continued. Blocked on an outgoing port indicates that the subsequent tasks on the path are disabled and will never execute. To keep the configuration process sound, certain consistency between the configuration settings on incoming and outgoing ports is necessary. For example, a blocked outgoing port on a task will entail a blocked incoming port on the consecutive task(s).

\subsection{Steering Configurations through Questionnaires}

In [17] it is argued that the variability of a domain should be separated from the process model. This is to relieve the need for modeling expertise during the individualization of a configurable model for a specific context. Furthermore, it would be rather complex to evaluate the soundness and correctness of a process model and associated domain constraints restricting it at the same time. Therefore a mechanism for keeping a process model apart from the domain constrains associated to it is proposed in [17].

A domain questionnaire model contains a set of questions with related facts, both specified in natural language. The questions may be dependent on each other, hence the order in which they should be presented (or asked) to business users needs to be defined. The facts are boolean variables used to describe different features of a specific domain. They can be either disabled or enabled and they shape the space of all possible answers to a question. By answering a specific question the values of all facts related to it are set at once. A domain questionnaire model encapsulates all domain specific constraints needed to configure a general process model capturing the same domain.

To capture the relationship between process model and constraints, a restraints mapping using standard logical expressions is used. Apart from solving the interdependency issues it provides the model with a certain flexibility, making it possible to individualize a number of different process models for a given domain in an automated fashion without compromising the correctness of the domain or the process model. 


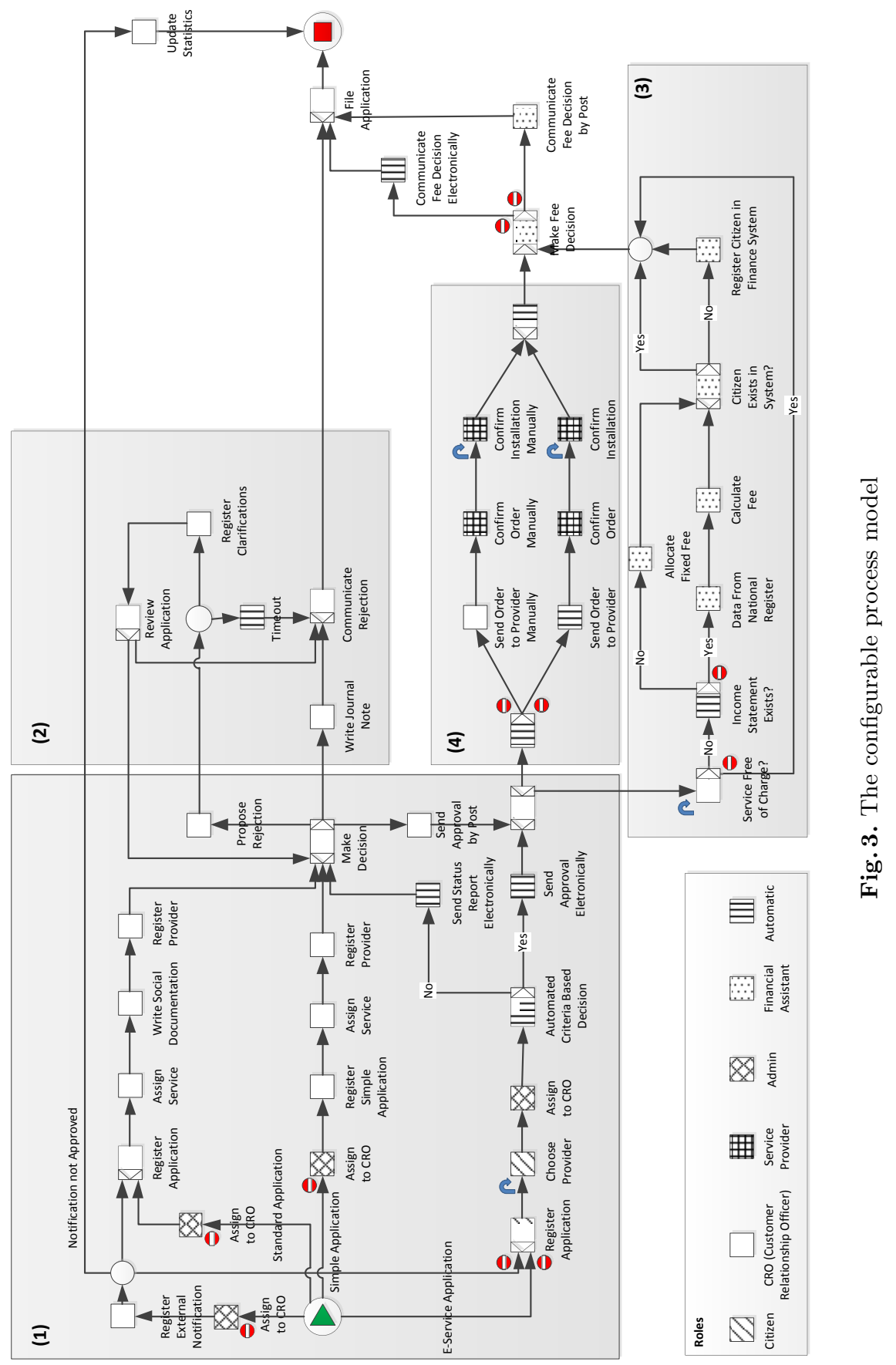




\section{The Configurable Process Model}

\subsection{Process Model}

In this work, a configurable model was created by merging three processes for handling of citizens applications for three social services namely part-time successor, companion service and emergency phone.

The part-time successor is a relief service for people who care for an elderly or disabled person. The companion service offers assistance to elderly or disabled people to participate in activities outside of their homes. These two social services are restricted to a certain amount of hours per month. They are payed by the subscribers based on the amount of utilized hours. The emergency phone service means that service subscribers can call for assistance $24 / 7$ if something unexpected happens which they can not deal with on their own. This service requires extra equipment in form of an alarm watch linked to a phone. The installation is taken care of by the service provider company. The service costs a fixed monthly fee.

These are social services, which means that they are sponsored by the local government. The role of the municipality is to determine which applicants are entitled for the services and how much they should pay (economically weak applicants are entitled to pay less). Also to ensure that the service subscriptions are allocated to the correct service provider and to follow up on the provision of the services are obligations carried out by the municipality. The applications for the services can come in three different forms, as an e-service application, as a simplified application or as a standard application. The underlying processes with their different variations are depicted in Figure 3 . Area (1) in this figure shows the three variants of receipt and registration of an application, as well as the decision made for an application. Positive decisions lead to two parallel treads of activities: contacting a service provider (Area (4) in the figure) and calculating and deciding on the service fee (most of the relevant activities in this part of the process are captured in Area (3) in the model). Finally, the activities associated to negative decisions are depicted in Area (2) in the model.

A common feature for the three e-services is that positive decisions are made automatically, based on a set of predefined criteria. The criteria are specific for each service and visible for the applicants. Some overlaps are observed between the criteria for the three services, but there are also differences. The intention with the predefined criteria is to increase the transparency towards the citizens as well as to filter and quickly handle the obviously positive decisions. Due to the availability of criteria and automated positive decisions, the services are called open social services. Negative decisions are never taken automatically.

The model was developed following the process modelling guidelines in 11. We have used as few elements as possible in the model but a few tasks have been duplicated. This was done to reduce the number of routing paths and to reinforce the comprehensiveness (clearness) of the model. 


\subsection{Domain Questionnaire Model}

To develop the domain questionnaire model for the case study we used the questionnaire designer tool in the Synergia toolset [15]. The model was derived by using material gathered from workshops and in depth interviews with representatives from all parts of the social services department at the municipality.

A companion service application is processed in the same fashion as a parttime successor application with the difference that the service is not offered free of charge, therefore a question mapping to this variation point is included in the domain questionnaire model. An emergency phone application is handled similarly, except that a provider for the service can not be chosen but is predefined, and thus a question regarding the possible choice of provider is also included in the domain questionnaire model. The service requires equipment installation, which is not needed in the other services and again this question exist in the domain questionnaire model. Furthermore, when citizens apply for emergency phone service through the e-service the fee is fixed and cannot be reduced. To capture this variation, there are two questions where one depends on the other in the domain questionnaire model. Besides these differences the municipality performs the same flow of activities for all three services. However, some questions that guide for example different type of services also have to be included in the domain questionnaire model. One of the individualized models, the emergency phone can be seen in Figure 5 .

The questionnaire model is depicted in Figure 4, green boxes represents domain questions, yellow ones represents the facts connected to a question and arrows imply that a dependency exist between questions. To relate the domain questionnaire and process configuration models a mapping between the two was performed in two steps: first the process model was used to map constraints into the domain questionnaire model and then the questions in the created domain questionnaire model were mapped to each variation point in the process model. These mappings were done manually as the mapping tool described in [17 for YAWL was released just after the study took place. Therefore the mapping verification functionality in the tool could not be utilized but instead the verification was carried out analytically.

\section{Evaluation}

The evaluation of the results was carried out through a case study, during which we validated the quality, generality and the perceived usefulness of the general model and the configuration approach.

\subsection{Quality}

The soundness of the configurable model was ensured through the verification functionality in YAWL. The ability of the model to capture the domain was ensured through input from the municipal officials and middle level management. 

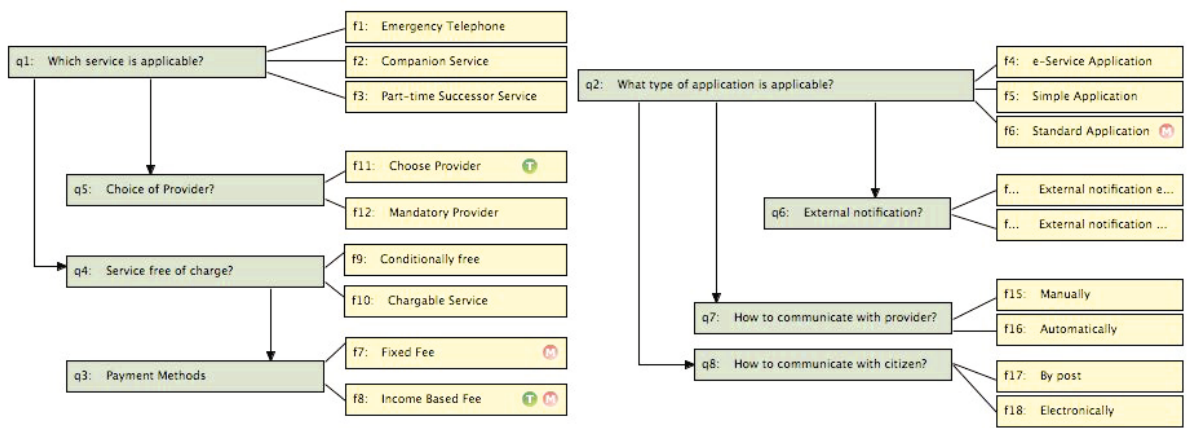

Fig. 4. Domain Questionnaire Model

To ensure quality in the received responses it was important to control that the respondents had a good understanding of the general model and the configuration of it. For this reason we used control question to check their understanding and we asked them to make suggestions for changes directly in the model. The respondents answered the control questions properly and also managed to apply suggested improvements directly in the model. They also asked very precise and logical questions e.g. "Is it possible to automate the Register Citizen in Finance System task?", "Make Fee Decision task has now been changed to be carried out by economy assistants!", "The task Write Social Documentation is done before Register Provider task" which showed they understood the model well.

\subsection{Generality}

The results from the study show that the configurable process model is general enough to cover a number of processes within the elderly and handicapped sector at Järfälla municipality. However, the model is not yet proven to be complete, as there is one additional process which we have not yet studied.

The generality of the model was evaluated by studying the extensibility of the model for accommodating a fourth process, i.e. the application handling process

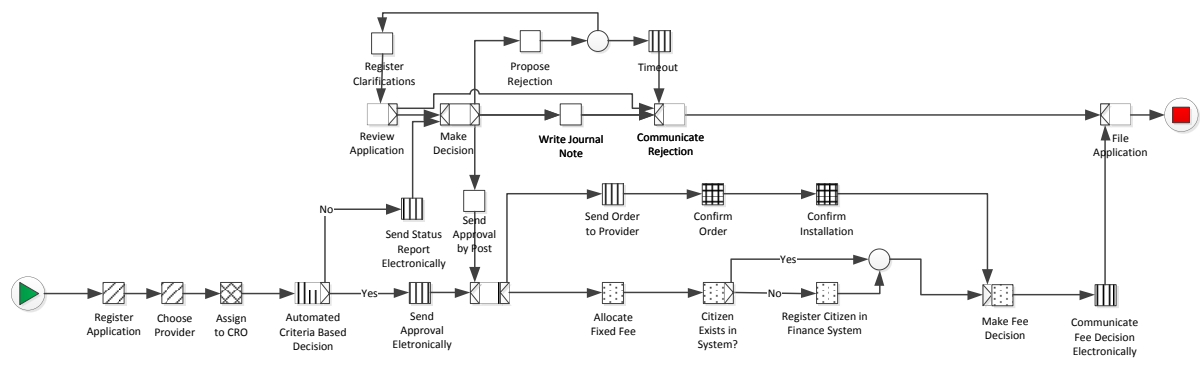

Fig. 5. The emergency phone e-service application - individualized process 
for home-care service. The application handling is similar to the handling of an emergency phone, part-time successor, and companion services applications. A difference is that the home-care service includes a number of different service efforts, e.g. cleaning, window-cleaning, laundry, grocery shopping and meal delivery. It is possible to apply for one or several of these service efforts in the same application, however different levels of granularity did not have to be considered due to the fact that the decision made is for the home-care service as a whole. It was concluded that neither the control flow nor the allocation of resources in the configurable model had do be adapted in order to accommodate the home-care service. The configurable model was general enough in those two perspectives to completely capture the new process. Modifications in the data perspective, to accommodate decision criteria specific for the home-care service and the choice of what specific service efforts to apply for were on the other hand needed. As a new process always implies new data that will have to be taken care of, this would not have been possible to avoid.

Regarding the completeness of the solution, it should be noted that the set of processes studied so far is not exclusive. To make the analysis exclusive, a fifth process needs to be added to the solution, namely the process of handling applications for the personal-care service. In contrast to the other services incorporated in the model so far, the municipality will not offer the personal-care service as an open service, i.e. automated positive decision making based on criteria will not be implemented for this service. This means that to provide the application of personal-care service as an e-service, the model in Figure 3 would need to be extended with a flow from the e-service application thread (bottom left corner in the model) to the manual "Make Decision" task in the middle of the model, and a hiding configuration would be added to the "Automated Criteria Based Decision" task. I.e. the configurable model could incorporate the new process with only minor modifications. This extension was discussed with the municipal officials in order to highlight the difference between open-services and e-services - two concepts that until now were used as synonyms by the municipality. In order to fully extend the solution for the personal-care service, the analysis for the data perspective need to be added. Nonetheless, the small impact on the model for incorporating the proposed extension from open e-services to e-services confirms the expected benefit on process change management (see Section [1).

To validate the questions in the domain model, we highlighted the configuration points and demonstrated the questions and how the answers individualized the configurable model. The individualization example we used was for the emergency phone e-service application, depicted in Figure 5. The questions got a good acceptance by the officials. They were considered as a natural way to capture the variations between the processes. This confirmed our expectation of easiness to produce configurations and support the envisaged individualization benefit discussed in Section 1 . 


\subsection{Perceived Usefulness}

The configurable process model is perceived useful since it promotes shared understanding and simplifies process analysis (see the discussion on the comprehensive process model benefit in Section 1). With regard to perceived usefulness, we distinguish between impressions from the officials that were earlier involved in the analysis of the three other application processes and the officials that were new in the project. The first group of officials emphasized that it was less timeconsuming to analyze the underlying process for home-care application then for the first three processes. The officials believed that this is due to the fact that the configurable process model served as a "great starting point". Another contributing factor to the timesavings was reuse of concepts and terms that had already been defined and built into the process model. The configurable process model was also appreciated by the officials new in the project. They valued the model because it gave them a good and comprehensive picture of the work routines and the rules in place.

To further test the perceived usefulness of the configurable model we asked the municipal officials to comment on the usefulness of the integrated process model. The immediate reaction from three officials was "It is nice to see such a flow describing what we actually do", "It gives a comprehensive picture of our work", and "It is good because our managers can see what we are doing". We also asked if the municipal officials would like to have posters with the processes, and if so which ones i.e. the general configurable process or the four configurations for the e-services. The answers from the working group were unanimous. The officials wanted all five models, i.e. both the general model and the four e-services configurations of it, as posters. This answer confirm the perceived usefulness of the configurable model for the social sector at Järfälla municipality.

\section{Challenges}

During our work we met a number of challenges that can be classified into the following areas: challenges related to the process modeling and execution paradigm, challenges associated to the configurable approach we have utilized, and challenges associated to the tools we used.

One of the main challenges related to process modeling and the execution paradigm during our work has been the difficulty in getting an understanding of the software support that a business process management system offers to a local government. At Järfälla municipality work has been made for streamlining the processes, however, the current (legacy) IT system at the municipality does not offer any support for these new processes. Therefore it is a challenge to reach an understanding of potential benefits in utilizing a process centric tool such as YAWL beyond that of being able to conceptualize and make the work processes visible to the municipal officials. The same difficulty has been observed regarding requirements specification for the e-services. Even though a validated process model with data and roles has been developed and used as requirements 
documentation towards the IT-service providers involved in the project, the process model has not been used as a blueprint for the actual development of the e-services it describes.

Challenges associated to the configuration approach mainly have to do with difficulties in capturing all variation points in the general model while at the same time maintaining an understandable and sound model. Since YAWL does not support configuration of data or resources we had to make workaround solutions that enlarged the model and duplicated a number of tasks. This was necessary in order to make the role configuration visible on a conceptual level. On the data level we made the decision of not capturing the configurations by multiplying tasks where data differed since this would have made the general model complex and difficult to understand. Also, since the municipality does not have a business process management system, the necessity of capturing data configurations in the model using a workaround solution was not considered as vital at this stage, although one of the most important arguments for choosing YAWL, that it is an executable notation, thereby became weaker. However, we still consider the executable feature of the YAWL notation an important argument for continuous use. The reason is that configuration on resource- and data level has already been solved conceptually [16] and only the implementation in the YAWL engine remains.

Challenges associated to the tools we used were mostly due to the fact that these are still at a prototype stage of development. For instance, apart from the configurable model capturing all variation points, we had to construct nine configurable models, one for each individualization we wanted to define. This was because the configuration feature of the YAWL editor only supports an on/off switch to apply all or none of the configurations made on a model. When the configuration is turned on all hides and blocks in the model are applied. Furthermore, the verification of models containing OR -splits and -joins is a known problem that is highlighted in [212. In order to use the verification functionality in YAWL to guarantee the correctness of the configurable model, we worked around all OR-splits and OR-joins, present in the model initially, by replacing them with corresponding constructs. By ensuring the correctness of the configurable model, we could guarantee the correctness of all individualized models extracted from it 1 .

\section{Where Next?}

Future work can be undertaken in two directions. The first line of work is to further extend the configurable model presented in this paper. The model now covers four processes in three different variants each. A beneficial extension would be to expand the general model to include e-services for processes dealing with the applications for personal-care services. As mentioned in Section 5] further configurations of resources and changes in the control-flow is expected to be minor. However a more detailed analysis of data may lead to more significant extensions of the process model, hence such extensions would deserve a dedicated 


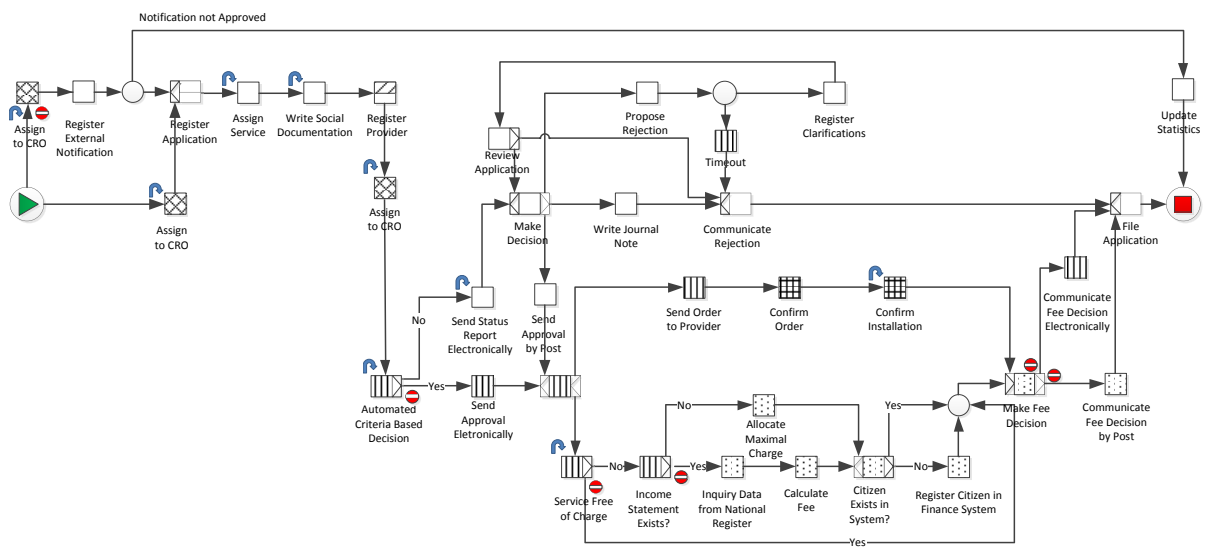

Fig. 6. The configurable process with data and resource configurations supported

analysis. Another useful extension would be the generalization of the model to cover the needs of several municipalities in the short term and capturing the social services application handling process at a national level in the long term. All municipalities in the country are obliged to provide their citizens with the same kind of services. They all need to handle similar kinds of administration work associated with the services and there are commonalities in the IT environments at different municipalities. For example approximately half of the municipalities in Sweden use the same IT-system as the municipality studied in this paper. The development of a general model for national use will enable knowledge sharing between municipalities and increase the transparency between local governments and citizens. A dedicated study (or a series of studies) including several municipalities is necessary to be carried out in order to address this issue.

The second line of work is further development of C-YAWL to also cover configuration of resource and data perspectives. The process model presented here was relatively small and the configuration of the resources could be dealt with by duplicating the tasks that could be executed by different resources. As discussed in the previous section, the same solution could have been applied to deal with the data configuration, however we postulated that this would compromise the clarity and the simplicity of the model. If configurations on the resource and data perspectives were supported in C-YAWL, the configurable process model developed here could be further simplified as depicted in Figure 6. While an approach for such configurations have already been proposed in [16] and developed for the C-iEPC notation, it has not yet been implemented in C-YAWL. An implementation in C-YAWL would be highly relevant for the Swedish public sector. By providing an executable solution we would be able to exemplify the full potential of the approach and work for increased awareness of the relationship between business processes, the process models capturing them, and the IT-systems supporting their execution. 


\section{Conclusion}

In this paper we have utilized a configurable process modeling approach, suggested in [4] and developed a general process model covering three different processes for the social services department at a municipality in Sweden. While this approach has earlier been used for integrating variants of one process for example in [5], the uniqueness of our work is that the approach has successfully been implemented to integrate a number of different but highly similar processes. We envisaged four potential benefits of developing a general model for the social services sector using a configurable approach.

The model and the approach have been evaluated in workshops where the understanding of the solution and benefits with it have been studied. Also a fourth process have been incorporated into the general model, which was possible without any changes to the control-flow and resource perspectives of the model. The evaluation shows that using configurable process models facilitate understanding and organizational change within the social services department. The general model is regarded as valuable for gaining a mutual understanding of how the work should be carried out and for educating new employees. Thereby the first benefit has been shown to be fulfilled. The use of questions that capture the domain restrictions applicable to the model has also been found valuable and is regarded as easily understood by the social services personnel wherefore we also consider the second benefit fulfilled.

During the evaluation work, and the incorporation of a fourth service into the general model, some legal changes has been observed. Also discussions on how to further utilize the electronic services by expanding their use to include services other than the open social e-services and the visualization of these envisioned changes into the general model points towards the third benefit being realistic, although the full advantage of it would be greater if a business process management software, supporting the processes, was used in the municipality.

Our work also shows that in order to take full advantage of the configurable approach, software support that enables the use of executable processes is needed. One of the main challenges identified is the old legacy systems used in the municipality, systems that do not support process orientation and are a great obstacle in making the social services enabled for e-government. The old IT-systems also work as a hurdle against the understanding of how IT can be a support for the local government. Today most social services officials consider IT more as a hindrance, slowing down and creating unnecessary frustration in their daily work. In our further work to realize the fourth benefit we expect this to be a challenge since the usage of old IT-systems is a reality in most municipalities in Sweden. The conclusion is thereby that out of the four envisaged benefits, two are considered fulfilled, the third considered realistic and the fourth needs further study in order to be proved.

A configurable process model can potentially become complex for example depending on the complexity of the domain and the number of variations points. It is also shown in 20] that the complexity of a configurable model is related to the similarity of the models that has been integrated to create the configurable 
model. If the dissimilarities between the integrated models are to many the configurable model becomes to complex to verify and validate. We have not seen any technical limitations of the configurable approach due to complex models, which might be due to the relatively small size (46 tasks) of the developed model. It could be argued that the generality of the study to cover larger and more complex models are questionable. However we consider it to be representative for two reasons: (1) The model makes use of all elements available in the configurable process approach. (2) The size of the model is not unusual if compared to other models collected from the public sector [20,5].

During our work with the configurable approach used in this study we have not recognized any concrete pitfalls or limitations affecting the feasibility of configurable models in the domain of the Swedish public sector. The question remains as to whether it is possible to incorporate a larger number of processes into the general model or if the dissimilarities between these models are so great that it becomes an obstacle? Our belief is that the developed configurable model will be able to cover a large share of processes within the public sector domain in Sweden.

Acknowledgement. We would like to thank all officials from Järfälla municipality who participated in the project for their deep involvement in the work.

\section{References}

1. van der Aalst, W.M.P., Dumas, M., Gottschalk, F., ter Hofstede, A.H.M., La Rosa, M., Mendling, J.: Correctness-Preserving Configuration of Business Process Models. In: Fiadeiro, J.L., Inverardi, P. (eds.) FASE 2008. LNCS, vol. 4961, pp. 46-61. Springer, Heidelberg (2008)

2. van der Aalst, W.M.P., Dumas, M., Gottschalk, F., ter Hofstede, A.H.M., La Rosa, M., Mendling, J.: Preserving correctness during business process model configuration. Formal Asp. Comput. 22(3-4), 459-482 (2010)

3. YAWL foundation. YAWL: Yet Another Workflow Language, www. yawlfoundation.org (accessed November 2011)

4. Gottschalk, F., van der Aalst, W.M.P., Jansen-Vullers, M.H., La Rosa, M.: Configurable Workflow Models. Int. J. Cooperative Inf. Syst. 17(2), 177-221 (2008)

5. Gottschalk, F., Wagemakers, T.A.C., Jansen-Vullers, M.H., van der Aalst, W.M.P., La Rosa, M.: Configurable Process Models: Experiences from a Municipality Case Study. In: van Eck, P., Gordijn, J., Wieringa, R. (eds.) CAiSE 2009. LNCS, vol. 5565, pp. 486-500. Springer, Heidelberg (2009)

6. Hallerbach, A., Bauer, T., Reichert, M.: Guaranteeing Soundness of Configurable Process Variants in Provop. In: Hofreiter, B., Werthner, H. (eds.) Prof. of IEEE Conference on Commerce and Enterprise Computing, pp. 98-105. IEEE Computer Society (2009)

7. Hevner, A.R., March, S.T., Park, J.: Design Science in Information Systems Research. MIS Quarterly 28(1), 75-106 (2004)

8. ter Hofstede, A.,van der Aalst W.M.P., Adams, M., Russell, N. (eds.): Modern Business Process Automation: YAWL and its Support Environment. Springer (2010) 
9. Juell-Skielse, G., Wohed, P.: Design of an Open Social E-Service for Assisted Living. In: Wimmer, M.A., Chappelet, J.-L., Janssen, M., Scholl, H.J. (eds.) EGOV 2010. LNCS, vol. 6228, pp. 289-300. Springer, Heidelberg (2010)

10. Dumas La Rosa, M., van der Aalst, W.M.P., ter Hofstede, A.H.M.: Questionnairebased Variability Modeling for System Configuration. Software and Systems Modeling (SoSyM) 8(2) (2009)

11. Mendling, J., Reijers, H.A., van der Aalst, W.M.P.: Seven process modeling guidelines (7pmg). Information \& Software Technology 52(2), 127-136 (2010)

12. Mendling, J., van Dongen, B.F., van der Aalst, W.M.P.: Getting Rid of the OR-Join in Business Process Models. In: 11th IEEE International Enterprise Distributed Object Computing Conference (EDOC 2007), pp. 3-14. IEEE Computer Society (2007)

13. Neale, P., Thapa, S., Boyce, C.: Prepareing A Case Study: A Guide for Designing and Conducting a Case Study for Evaluation Input. Pathfinder International (May 2006) (accessed November 2011)

14. Razavian, M., Khosravi, R.: Modeling Variability in Business Process Models Using UML. In: Proc. of 5th Int. Conf. on Information Technology: New Generations. IEEE Computer Society (2008)

15. La Rosa, M.: Process Configuration.com, www.processconfiguration.com (accessed November 2011)

16. La Rosa, M., Dumas, M., ter Hofstede, A.H.M., Mendling, J., Gottschalk, F.: Beyond Control-Flow: Extending Business Process Configuration to Roles and Objects. In: Li, Q., Spaccapietra, S., Yu, E., Olivé, A. (eds.) ER 2008. LNCS, vol. 5231, pp. 199-215. Springer, Heidelberg (2008)

17. La Rosa, M., Lux, J., Seidel, S., Dumas, M., ter Hofstede, A.H.M.: Questionnairedriven Configuration of Reference Process Models. In: Krogstie, J., Opdahl, A.L., Sindre, G. (eds.) CAiSE 2007. LNCS, vol. 4495, pp. 424-438. Springer, Heidelberg (2007)

18. Schnieders, A., Puhlmann, F.: Variability Mechanisms in E-Business Process Families. In: Abramowicz, W., Mayr, H.C. (eds.) Proc. of. 9th Int. Conf. on Business Information Systems. LNI, vol. 85, pp. 583-601. GI (2006)

19. Statistics Sweden. The future population of Sweden (2009), www.scb.se/statistik/_publikationer/BE0401_2009I60_BR_BE51BR0901ENG.pdf (accessed July 15 2010)

20. Vogelaar, J., Verbeek, H.M.W., Luka, B., van der Aalst, W.M.P.: Comparing Business Processes to Determine the Feasibility of Configurable Models: A Case Study, pp. 50-61 (2011) 